\title{
Molecular Interactions of Benzaldehyde with Benzene at 303.15, 308.15 and 313.15 K and a Pressure of 0.1 MPa
}

\author{
K. SARAVANAKUMAR ${ }^{\mathrm{a}^{*}}$, T. G. LAVANYA ${ }^{\mathrm{b}}$ and T. R. KUBENDRAN ${ }^{\mathrm{c}}$
}

${ }^{a}$ Department of Chemical Engineering, Sathyabama University, Jeppiar Nagar, Chennai-600119, India

${ }^{\mathrm{b}}$ Department of Chemical Engineering, Sri Venkateswara College of Engineering, Sriperumpudur, Chennai. India

${ }^{c}$ Department of Chemical Engineering, A.C. College of Technology, Anna University, Chennai-600025, India

saroo_1978@yahoo.co.in

Received 11 May 2012 / Accepted 28 May 2012

\begin{abstract}
The values of density, viscosity and speed of sound for the binary liquid mixture of benzaldehyde with benzene have been measured over the entire range of composition at 303.15, 308.15 and $313.15 \mathrm{~K}$. These values have been used to calculate the excess molar volume $\left(\mathrm{V}^{\mathrm{E}}\right)$, deviation in viscosity $(\Delta \eta)$, deviation in speed of sound $(\Delta U)$, deviation in isentropic compressibility $\left(\Delta \beta_{\mathrm{s}}\right)$, excess internal pressure $(\Delta \pi)$, excess intermolecular free length $\left(\Delta \mathrm{L}_{\mathrm{f}}\right)$, excess free volume $\left(V_{f}{ }^{\mathrm{E}}\right)$ and excess acoustic impedance $(\Delta \mathrm{Z})$. McAllister's three body interaction model is used for correlating kinematic viscosity of binary mixtures. The excess values were correlated using the Redlich-Kister polynomial equation to obtain their coefficients and standard deviations. The thermophysical properties (density, viscosity and speed of sound) under the study were fit to the Jouyban-Acree model.
\end{abstract}

Keywords: Molecular interaction, Density, Viscosity, Speed of sound

\section{Introduction}

The thermodynamic and transport properties of liquids and liquid mixtures ${ }^{1}$ are used to study the molecular interactions between the various components of the mixtures the various components of the mixture and also to understand engineering applications concerning heat transfer, mass transfer and fluid flow. In chemical process industries, materials are normally handled in fluid form and as a consequence, the physical, chemical and transport properties of fluids, assume importance. Thus, data on some of the properties associated with the liquids and liquid mixtures like Density, viscosity, refractive index and Speed of Sound find extensive application in solution theory and molecular dynamics. Such results are necessary 
for interpretation of data obtained from thermo chemical, electrochemical, biochemical and kinetic studies ${ }^{2}$. Benzene is used in the production of drugs. Benzoin prepared from Benzaldehyde is used as "tincture benzoin" in medicine for throat infection. In our earlier paper $^{3-5}$, we had studied the transport properties of binary liquid mixtures. In the present paper the densities, viscosities and ultrasonic velocities for the binary liquid mixtures of benzaldehyde with benzene have been measured over the entire range of composition at $303.15,308.15$ and $313.15 \mathrm{~K}$. To study the physicochemical behaviour of benzaldehyde with benzene by evaluating different thermo acoustic parameters like acoustical impedance $(Z)$,isentropic compressibility $\left(\beta_{\mathrm{s}}\right)$, intermolecular free length $\left(\mathrm{L}_{\mathrm{f}}\right)$, free volume $\left(\mathrm{V}_{\mathrm{f}}\right)$, degree of intermolecular attraction $(\alpha)$ and their excess properties were derived over the entire mol fraction range. The viscosity values have been fitted to McAllister ${ }^{6}$ and Krishnan and Laddha $^{7}$ model. The Jouyban - Acree model $^{8}$ has also been extended to density, viscosity and Speed of Sound of binary mixtures. The deviation values have been fitted to RedlichKister type ${ }^{9}$ equation. Literature survey showed that no measurements have been previously reported for the mixture studied in this paper.

\section{Experimental}

Benzaldehyde and benzene (Lobo Chemicals, India and mass fraction $\geq 0.998$ ) were purified by using the methods described in the literature ${ }^{10}$ and only middle fractions were used in the experiment. Binary mixtures are prepared by mixing appropriate volumes of the liquid components in the specially designed glass bottles with air tight Teflon coated caps and mass measurements performed on a Shimadzu Corporation Japan type BL 2205 electronic balance, with a precision of $\pm 0.01 \mathrm{mg}$. The required properties are measured on the same day immediately after preparing each composition. The uncertainty of the mole fraction is \pm 0.001 . The experimental values of density, viscosity and Speeds of Sound for the pure liquids are compared, with the available literature values at $\mathrm{T}=303.15 \mathrm{~K}$ in Table 1.

Table 1. Comparison of experimental density, $\rho$ and viscosity, $\eta$ and speed of sound of pure liquids with literature values at $308.15 \mathrm{~K}$

\begin{tabular}{|c|c|c|c|c|c|c|c|}
\hline \multirow{2}{*}{ Component } & \multirow{2}{*}{$\mathrm{T} / \mathrm{K}$} & \multicolumn{2}{|c|}{$\rho /($ kg.m-3) } & \multicolumn{2}{|c|}{$\eta \times 10^{3} \mathrm{~kg} \cdot \mathrm{m}^{-1} \mathrm{~s}^{-1}$} & \multicolumn{2}{|c|}{$\mathrm{u}(\mathrm{m} / \mathrm{s})$} \\
\hline & & Exp & Lit & Exp & Lit & Exp & Lit \\
\hline $\begin{array}{l}\text { Benzaldehyde } \\
\text { Benzene }\end{array}$ & $308.15 \mathrm{~K}$ & $\begin{array}{l}1.0313 \\
1.4747\end{array}$ & $\begin{array}{l}1.0335^{13} \\
1.4753^{15}\end{array}$ & $\begin{array}{l}1.2486 \\
1.1159\end{array}$ & $\begin{array}{c}1.2441^{14} \\
0.964^{15}\end{array}$ & $\begin{array}{l}1442^{17} \\
1122^{17}\end{array}$ & $\begin{array}{l}1464 \\
1121\end{array}$ \\
\hline
\end{tabular}

Apparatus and procedure

A double- arm pycnometer with a bulb of $25 \mathrm{~cm}^{3}$ and a capillary of an internal diameter of about $1 \mathrm{~mm}$ is used to measure the densities $(\rho)$ of pure liquids and binary mixtures. The pycnometer is calibrated by using conductivity water (having specific conductance less than $\left.1.10^{-6} \mathrm{ohm}^{-1}\right)$ with 0.9970 and $0.9940 \mathrm{gcm}^{-3}$ as its densities at $\mathrm{T}=(298.15$ and 308.15$) \mathrm{K}$, respectively. The pycnometer filled with air bubbles free liquids is kept in a thermostat with a thermal stability of $\pm 0.01 \mathrm{~K}$ for over $30 \mathrm{~min}$ to attain thermal equilibrium. The precision of the density measurements was estimated to be $\pm 0.002 \mathrm{~g} \cdot \mathrm{cm}^{-3}$.

The kinematic viscosities were measured with Ostwald viscometer previously calibrated using water. The time was measured with a precision of $0.01 \mathrm{~s}$ and the uncertainty in the viscosity was estimated to be less than $0.0002 \mathrm{mPa} \cdot \mathrm{s}$. The kinematic viscosity was obtained from the working equation

$$
v=a t-b / t
$$

Where the two constants $a$ and $b$ were obtained by measuring the flow time $t$ of benzene. 
Speed of sound was measured by using a variable path, single crystal interferometer (Mittal Enterprises, New Delhi) at a frequency of $2 \mathrm{MHz}$ and calibrated with toluene. The interferometer cell was filled with the test liquid and the temperature of the solution was maintained constant within $(0.01) \mathrm{K}$ by circulation of water from a thermostatically regulated water bath through the water jacketed cell. The uncertainty was estimated to be $2 \mathrm{~m} / \mathrm{s}$.

\section{Results and Discussion}

The viscosities of mixtures of benzaldehyde and benzene have been correlated with the model proposed by McAllister for a binary mixture. Considering three-body interaction ${ }^{3}$,

$$
\begin{gathered}
\ln v=x_{1}{ }^{3} \ln v_{1}+3 x_{1}{ }^{2} x_{2} \ln v_{12}+3 x_{1} x_{2}{ }^{2} \ln v_{21}+x_{2}{ }^{3} \ln v_{2}-\ln \left(x_{1}+x_{2} M_{2} M_{1}\right)+3 x_{1}{ }^{2} x_{2} \\
\ln \left(\left(2+M_{2} M_{1}\right) / 3\right)+x_{2}{ }^{3} \ln \left(M_{2} / M_{1}\right)+3 x_{1} x_{2}{ }^{2} \ln \left(\left(1+2 M_{2} / M_{1}\right) / 3\right)
\end{gathered}
$$

In equation $2, v_{1}$ and $v_{2}$ refer to the kinematic viscosity of pure liquids 1 and 2 having mole fractions $x_{1}$ and $x_{2}$, respectively. The parameters $v_{12}$ and $v_{21}$ represent the interaction parameters obtained by multiple regression analysis, while $M_{1}$ and $M_{2}$ are the molar mass of the components. The kinematic viscosity was correlated by means of the Krishnan and Laddha model $^{7}$ for a binary mixture, which gives.

$$
\ln v=\mathrm{x}_{1} \ln v_{1}+\mathrm{x}_{2} \ln v_{2}+\mathrm{x}_{1} \ln \mathrm{M}_{1}+\mathrm{x}_{2} \ln \mathrm{M}_{2}+\ln \left(\mathrm{x}_{1} \mathrm{M}_{1}+\mathrm{x}_{2} \mathrm{M}_{2}-2.30 \mathrm{x}_{1} \mathrm{x}_{2}\left(\mathrm{~B}+\mathrm{C}\left(\mathrm{x}_{1}-\mathrm{x}_{2}\right) \ldots\right)\right)
$$

Where $B$ and $C$ are interaction parameters. Jouyban et. al proposed a model ${ }^{8}$ for correlating the viscosity of liquid mixtures at various temperatures

$$
\ln \mathrm{y}_{\mathrm{m}}, \mathrm{T}=\mathrm{f}_{1} \ln \mathrm{y}_{1}+\mathrm{f}_{2} \ln \mathrm{y}_{2}+\mathrm{f}_{1} \mathrm{f}_{2} \Sigma\left[\mathrm{A}_{\mathrm{J}}\left(\mathrm{f}_{1}-\mathrm{f}_{2}\right)^{\mathrm{J}} / \mathrm{T}\right]
$$

Where $y_{m}, y_{1}, T$ and $y_{2, T}$ are the viscosity of the mixture and solvents 1 and 2 at temperature $T$, respectively. $A_{J}$ is the model constant. The densities $(\rho)$, viscosities $(\eta)$ and speed of sound $(\mathrm{u})$ for the binary mixtures at $\mathrm{T}=(303.15,308.15$ and 313.15$) \mathrm{K}$ is listed in

\begin{tabular}{|c|c|c|c|c|c|c|c|c|c|}
\hline $\mathrm{x}_{1}$ & $\begin{array}{l}\rho \times 10^{-3} \\
\mathrm{~kg} \cdot \mathrm{m}^{-3}\end{array}$ & $\begin{array}{c}\eta \times 10^{3} \\
\mathrm{~kg} \cdot \mathrm{m}^{-1} \mathrm{~s}^{-1}\end{array}$ & $\begin{array}{c}\mathrm{U} \\
\mathrm{m} \cdot \mathrm{s}^{-1}\end{array}$ & $\begin{array}{l}\rho \times 10^{-3} \\
\mathrm{~kg} \cdot \mathrm{m}^{-3}\end{array}$ & $\begin{array}{c}\eta \times 10^{3} \\
\mathrm{~kg} \cdot \mathrm{m}^{-1} \mathrm{~s}^{-1}\end{array}$ & $\begin{array}{c}\mathrm{U} \\
\mathrm{m} \cdot \mathrm{s}^{-1}\end{array}$ & $\begin{array}{l}\rho \times 10^{-3} \\
\mathrm{~kg} \cdot \mathrm{m}^{-3}\end{array}$ & $\begin{array}{c}\eta \times 10^{3} \\
\mathrm{~kg} \cdot \mathrm{m}^{-1} \mathrm{~s}^{-1}\end{array}$ & $\begin{array}{c}\mathrm{U} \\
\mathrm{m} \cdot \mathrm{s}^{-1}\end{array}$ \\
\hline & & $303.15 \mathrm{~K}$ & & & $08.15 \mathrm{~K}$ & & & $313.15 \mathrm{~K}$ & \\
\hline 0 & 0.8680 & 0.585844 & 1281 & 0.86196 & 0.57830 & 1253 & 0.85928 & 0.533773 & 1232 \\
\hline 0.0896 & 0.8756 & 0.62378 & 1294 & 0.87256 & 0.62627 & 1265 & 0.8715 & 0.592877 & 1242 \\
\hline 0.1813 & 0.8914 & 0.665918 & 1305 & 0.8898 & 0.66357 & 1278 & 0.8879 & 0.650551 & 1255 \\
\hline 0.2751 & 0.9148 & 0.712648 & 1317 & 0.91396 & 0.70956 & 1291 & 0.9129 & 0.699998 & 1270 \\
\hline 0.3712 & 0.9406 & 0.775173 & 1330 & 0.9392 & 0.77231 & 1305 & 0.9375 & 0.757728 & 1285 \\
\hline 0.4697 & 0.9612 & 0.840553 & 1344 & 0.9582 & 0.83539 & 1321 & 0.9569 & 0.820839 & 1302 \\
\hline 0.5705 & 0.9765 & 0.910689 & 1360 & 0.9744 & 0.90281 & 1339 & 0.9728 & 0.889263 & 1320 \\
\hline 0.6739 & 0.9894 & 0.987915 & 1376 & 0.9883 & 0.98161 & 1357 & 0.9865 & 0.966833 & 1339 \\
\hline 0.7799 & 0.9989 & 1.070894 & 1394 & 0.9982 & 1.06759 & 1376 & 0.9963 & 1.062935 & 1358 \\
\hline 0.8885 & 1.0126 & 1.173349 & 1415 & 1.011 & 1.16619 & 1397 & 1.0095 & 1.159167 & 1379 \\
\hline 1 & 1.0318 & 1.259898 & 1442 & 1.03135 & 1.24886 & 1428 & 1.02804 & 1.241353 & 1413 \\
\hline
\end{tabular}
Table 2.

Table 2. Densities $\rho$, viscosities $\eta$, Speeds of Sound u for the benzaldehyde(1)+benzene(2) mixture at $\mathrm{T}=(303.15,308.15$ and 313.15$) \mathrm{K}$

The excess properties $\mathrm{A}^{\mathrm{E}}$ that shed light on the deviation from ideality are responsible for assessing the structural variation and the type of molecular interactions. Acoustical impedance $(Z)$, isentropic compressibility $\left(\beta_{\mathrm{s}}\right)$, intermolecular free length $\left(\mathrm{L}_{\mathrm{f}}\right)$, intermolecular attraction $(\alpha)$ and Internal pressure $(\pi)$ can be calculated using the following relations and presented in Table 3-5. 
Table 3. Acoustical impedance $(\mathrm{Z})$, isentropic compressibility $(\beta \mathrm{s})$, Internal pressure $(\pi \mathrm{i})$,intermolecular free length (Lf), Inter molecular attraction $(\alpha) \mathrm{T}=303.15 \mathrm{~K}$

\begin{tabular}{ccccccc}
\hline $\mathrm{X}_{1}$ & $\begin{array}{c}\mathrm{Z} \times 10^{-5} \\
\mathrm{~kg} \cdot \mathrm{m}^{-2} \cdot \mathrm{s}^{-1}\end{array}$ & $\begin{array}{c}\beta \mathrm{s} \times 10^{10} \\
\mathrm{~Pa}^{-1}\end{array}$ & $\begin{array}{c}\pi_{\mathrm{i}} \times 10^{-5} \\
\mathrm{~N}^{-2} \mathrm{~m}^{-2}\end{array}$ & $\begin{array}{c}\mathrm{L}_{\mathrm{f}} \times 10^{11} \\
\mathrm{~m}\end{array}$ & $\begin{array}{c}\mathrm{V}_{\mathrm{f}} \\
\mathrm{m}^{3} \mathrm{~mol}^{-1}\end{array}$ & $\alpha$ \\
\hline 0 & 11.1242 & 7.01415 & 1.25773 & 5.29684 & 0.00796 & 0 \\
0.0896 & 11.3302 & 6.82064 & 1.25184 & 5.22327 & 0.00771 & 0.01281 \\
0.1813 & 11.6322 & 6.58757 & 1.25636 & 5.13325 & 0.00742 & 0.02060 \\
0.2751 & 12.0479 & 6.30234 & 1.26922 & 5.02089 & 0.00712 & 0.02677 \\
0.3712 & 12.5094 & 6.01049 & 1.29417 & 4.90326 & 0.00667 & 0.03093 \\
0.4697 & 12.9185 & 5.75953 & 1.31214 & 4.79980 & 0.00628 & 0.03260 \\
0.5705 & 13.2804 & 5.53668 & 1.32397 & 4.70603 & 0.00594 & 0.03278 \\
0.6739 & 13.6230 & 5.33117 & 1.33431 & 4.61786 & 0.00560 & 0.02914 \\
0.7799 & 13.9238 & 5.15203 & 1.34125 & 4.53961 & 0.00529 & 0.01993 \\
0.8885 & 14.3282 & 4.93229 & 1.35773 & 4.44175 & 0.00494 & 0.00969 \\
1 & 14.8779 & 4.66112 & 1.36272 & 4.31792 & 0.00477 & 0 \\
\hline
\end{tabular}

Table 4. Acoustical impedance $(Z)$, isentropic compressibility $(\beta s)$, Internal pressure $(\pi \mathrm{i})$, intermolecular free length $\left(\mathrm{L}_{\mathrm{f}}\right)$, Inter molecular attraction $(\alpha) \mathrm{T}=308.15 \mathrm{~K}$

\begin{tabular}{ccccccc}
\hline $\mathrm{X}_{1}$ & $\begin{array}{c}\mathrm{Zx10} 0^{-5} \\
\mathrm{~kg} \cdot \mathrm{m}^{-2} \cdot \mathrm{s}^{-1}\end{array}$ & $\begin{array}{c}\beta \mathrm{s} \times 10^{10} \\
\mathrm{~Pa}^{-1}\end{array}$ & $\begin{array}{c}\pi_{\mathrm{i}} \times 10^{-5} \\
\mathrm{~N}^{-2} \mathrm{~m}^{-2}\end{array}$ & $\begin{array}{c}\mathrm{L}_{\mathrm{f}} \times 10^{11} \\
\mathrm{~m}\end{array}$ & $\begin{array}{c}\mathrm{V}_{\mathrm{f}} \\
\mathrm{m}^{3} \mathrm{~mol}^{-1}\end{array}$ & $\alpha$ \\
\hline 0 & 10.8003 & 7.38942 & 1.27868 & 5.43669 & 0.00784 & 24.08 \\
0.0896 & 11.0378 & 7.16182 & 1.28659 & 5.35231 & 0.00740 & 24.73 \\
0.1813 & 11.3716 & 6.88091 & 1.28673 & 5.24629 & 0.00723 & 25.61 \\
0.2751 & 11.7992 & 6.56478 & 1.29947 & 5.12436 & 0.00695 & 26.70 \\
0.3712 & 12.2565 & 6.25202 & 1.32433 & 5.00081 & 0.00652 & 27.89 \\
0.4697 & 12.6626 & 5.97598 & 1.33817 & 4.88916 & 0.00618 & 28.99 \\
0.5705 & 13.0472 & 5.72402 & 1.34852 & 4.78498 & 0.00588 & 30.07 \\
0.6739 & 13.4112 & 5.49479 & 1.36087 & 4.68819 & 0.00554 & 31.12 \\
0.7799 & 13.7352 & 5.29109 & 1.36957 & 4.60047 & 0.00521 & 32.09 \\
0.8885 & 14.1236 & 5.06822 & 1.38330 & 4.50254 & 0.00489 & 33.25 \\
1 & 14.7359 & 4.74953 & 1.38511 & 4.35868 & 0.00477 & 35.09 \\
\hline
\end{tabular}

Table 5. Acoustical impedance $(Z)$, isentropic compressibility $(\beta s)$, Internal pressure $(\pi \mathrm{i})$, intermolecular free length $\left(\mathrm{L}_{\mathrm{f}}\right)$, Inter molecular attraction $(\alpha) \mathrm{T}=313.15 \mathrm{~K}$

\begin{tabular}{ccccccc}
\hline $\mathrm{X}_{1}$ & $\begin{array}{c}\mathrm{Zx10} 0^{-5} \\
\mathrm{~kg} \cdot \mathrm{m}^{-2} \cdot \mathrm{s}^{-1}\end{array}$ & $\begin{array}{c}\beta \mathrm{s} \times 10^{10} \\
\mathrm{~Pa}^{-1}\end{array}$ & $\begin{array}{c}\pi_{\mathrm{i}} \times 10^{-5} \\
\mathrm{~N} \cdot \mathrm{m}^{-2}\end{array}$ & $\begin{array}{c}\mathrm{L}_{\mathrm{f}} \times 10^{11} \\
\mathrm{~m}\end{array}$ & $\begin{array}{c}\mathrm{V}_{\mathrm{f}} \\
\mathrm{m}^{3} \mathrm{~mol}^{-1}\end{array}$ & $\alpha$ \\
\hline 0 & 10.5897 & 7.66234 & 1.25618 & 5.5361 & 0.00863 & 0 \\
0.0896 & 10.8240 & 7.43856 & 1.28282 & 5.4547 & 0.00782 & 0.00702 \\
0.1813 & 11.1431 & 7.15069 & 1.30468 & 5.3481 & 0.00724 & 0.01646 \\
0.2751 & 11.5938 & 6.79155 & 1.32141 & 5.2121 & 0.00692 & 0.02566 \\
0.3712 & 12.0468 & 6.45985 & 1.34178 & 5.0832 & 0.00655 & 0.03090 \\
0.4697 & 12.4588 & 6.16469 & 1.35682 & 4.9657 & 0.00621 & 0.03484 \\
0.5705 & 12.8409 & 5.89968 & 1.36834 & 4.8578 & 0.00588 & 0.03522 \\
0.6739 & 13.2092 & 5.65381 & 1.38002 & 4.7555 & 0.00555 & 0.03131 \\
0.7799 & 13.5297 & 5.44264 & 1.39617 & 4.6658 & 0.00515 & 0.02072 \\
0.8885 & 13.9260 & 5.20535 & 1.40898 & 4.5630 & 0.00484 & 0.00627 \\
1 & 14.5262 & 4.87198 & 1.40816 & 4.4145 & 0.00474 & 0 \\
\hline
\end{tabular}


The acoustical impedance The isentropic compressibility Intermolecular free length Intermolecular attraction

$$
\begin{aligned}
& \mathrm{Z}=\rho \mathrm{u} \\
& \beta_{\mathrm{s}}=1 / \rho \mathrm{u}^{2} \\
& \mathrm{~L}_{\mathrm{f}}=\mathrm{K} \beta_{\mathrm{s}}^{1 / 2} \\
& \alpha=\left(\mathrm{u}^{2} / \mathrm{u}_{\mathrm{im}}^{2}\right)-1
\end{aligned}
$$

Where $\mathrm{u}_{\text {im }}^{2}=1 /\left\{\left(x_{1} \mathrm{M}_{1}+x_{2} \mathrm{M}_{2}\right)\left(x_{1} / \mathrm{M}_{1} \mathrm{u}_{1}^{2}+x_{2} / \mathrm{M}_{2} \mathrm{u}_{2}^{2}\right)\right\}$

Internal pressure

$$
\begin{aligned}
\pi=\operatorname{bRT}(\mathrm{K \eta} / \mathrm{U}) \stackrel{1 / 2}{2}^{2 / 3}\left(\rho^{7 / 6} / \mathrm{M}_{\text {eff }}^{7 / 6}\right) \\
V_{f}=\left(M_{\text {eff }} U / \eta k\right)^{3 / 2}
\end{aligned}
$$

Where $\mathrm{M}$ is the molar mass of mixture and $k$ is a constant equal to $4.28 \times 10^{9}, \mathrm{M}_{1}$ and $\mathrm{M}_{2}$ are the molar mass of component 1 and 2 respectively. All the excess and deviation quantities $\left(\mathrm{V}^{\mathrm{E},} \Delta \eta, \Delta \mathrm{u}, \Delta \beta_{\mathrm{s},} \Delta \mathrm{L}_{\mathrm{f},}, \mathrm{V}_{\mathrm{f}}{ }^{\mathrm{E}}\right.$ and $\left.\Delta \mathrm{Z}\right)$ have been fitted to the Redlich-Kister ${ }^{6}$ polynomial equation given below:

$$
\mathrm{A}^{\mathrm{E}}=\mathrm{x}_{1} \mathrm{x}_{2} \Sigma \mathrm{Ai}\left(\mathrm{x}_{1}-\mathrm{x}_{2}\right)^{\mathrm{I}}
$$

Where $\mathrm{N}$ is the number of data points and $\mathrm{n}$ is the number of coefficients, $A_{i}$ is polynomial coefficients obtained by fitting equation.12 to experimental data using a least-squares regression method. The standard deviations (S) were calculated using the relation. The calculated values of coefficients along with the standard deviation $(S)$ are given in Table.6-13.

$$
\mathrm{S}=\left\{\Sigma(\text { Aexp }- \text { Acal })^{2} /(\mathrm{N}-\mathrm{n})\right\}^{1 / 2}
$$

Table 6. Parameters of the McAllister model and standard deviations for benzaldehyde (1) +benzene (2) at $\mathrm{T}=(303.15,308.15$ and 313.15$) \mathrm{K}$

\begin{tabular}{cccc}
\hline \multirow{2}{*}{$\mathrm{T} / \mathrm{K}$} & \multicolumn{3}{c}{ Benzaldehyde+Benzene } \\
\cline { 2 - 4 } & $\mathrm{A}$ & $\mathrm{B}$ & $\mathrm{S}$ \\
\hline 303.15 & 1.01594 & 0.78936 & 0.01378 \\
308.15 & 1.0049 & 0.8008 & 0.019983 \\
313.15 & 0.969713 & 0.83074 & 0.040981 \\
\hline
\end{tabular}

Table 7. Parameters of the Krishnan and Laddha model and standard deviations $\mathrm{S}$ for the viscosity of benzaldehyde (1) +benzene (2) at $\mathrm{T}=(303.15,308.15$ and 313.15$) \mathrm{K}$

\begin{tabular}{cccccc}
\hline $\mathrm{T} / \mathrm{K}$ & $\mathrm{A}_{0}$ & $\mathrm{~A}_{1}$ & $\mathrm{~A}_{2}$ & $\mathrm{~A}_{3}$ & $\mathrm{~S}$ \\
\hline 303.15 & -0.0883 & 0.093 & -0.0176 & 0.0086 & 0.00365 \\
308.15 & 0.0747 & 0.221 & -0.0447 & -0.001 & 0.00575 \\
313.15 & 0.1185 & -0.014 & 0.0209 & -0.0367 & 0.00331 \\
\hline
\end{tabular}

Table 8. Parameters of the Jouyban-Acree model and standard deviations $\mathrm{S}$ for the viscosity of benzaldehyde (1) +benzene (2) at $\mathrm{T}=(303.15,308.15$ and 313.15$) \mathrm{K}$

\begin{tabular}{cccccc}
\hline $\mathrm{T} / \mathrm{K}$ & $\mathrm{A}_{0}$ & $\mathrm{~A}_{1}$ & $\mathrm{~A}_{2}$ & $\mathrm{~A}_{3}$ & $\mathrm{~S}$ \\
\hline $303.15 \mathrm{~K}$ & 75.116 & -57.948 & -4.136 & 25.308 & 0.06412 \\
$308.15 \mathrm{~K}$ & 52.747 & -42.628 & -4.186 & 27.913 & 0.00082 \\
$313.15 \mathrm{~K}$ & 44.358 & -57.96 & -3.926 & 30.18 & 0.00121 \\
\hline
\end{tabular}

Table 9. Parameters of the Jouyban-Acree model and standard deviations $\mathrm{S}$ for the density of benzaldehyde (1) +benzene (2) at $\mathrm{T}=(303.15,308.15$ and 313.15$) \mathrm{K}$

\begin{tabular}{cccccc}
\hline $\mathrm{T} / \mathrm{K}$ & $\mathrm{A}_{0}$ & $\mathrm{~A}_{1}$ & $\mathrm{~A}_{2}$ & $\mathrm{~A}_{3}$ & $\mathrm{~S}$ \\
\hline 303.15 & -176.41 & 16.453 & 4.5924 & -52.09 & 0.7053 \\
308.15 & -232.1 & 73.447 & 14.392 & -59.87 & 1.4236 \\
$\mathbf{3 1 3 . 1 5}$ & -183.27 & 110.59 & 12.377 & -65.21 & 1.4410 \\
\hline
\end{tabular}


Table 10. Parameters and standard deviations of the Jouyban-Acree model for the speed of sound of benzaldehyde (1) +benzene (2) at $\mathrm{T}=(303.15,308.15$ and 313.15$) \mathrm{K}$

\begin{tabular}{cccccc}
\hline $\mathrm{T} / \mathrm{K}$ & $\mathrm{A}_{0}$ & $\mathrm{~A}_{1}$ & $\mathrm{~A}_{2}$ & $\mathrm{~A}_{3}$ & $\mathrm{~S}$ \\
\hline 303.15 & 142.6 & -110.68 & 8.636 & 4.7942 & 0.00433 \\
308.15 & 5.767 & -187.18 & 28.01 & 13.851 & 0.00564 \\
313.15 & -34.9 & -35.717 & -18.47 & 41.654 & 0.00182 \\
\hline
\end{tabular}

Table 11. Parameters of Redlich-Kister equation and standard deviations for benzaldehyde (1) + Benzene (2) $\mathrm{T}=303.15 \mathrm{~K}$

\begin{tabular}{lccccc}
\hline \multicolumn{1}{c}{ Functions } & $\mathrm{A}_{0}$ & $\mathrm{~A}_{1}$ & $\mathrm{~A}_{2}$ & $\mathrm{~A}_{3}$ & $\mathrm{~S}$ \\
\hline $\mathrm{V}^{\mathrm{E}} \cdot 10^{6} / \mathrm{m}^{3} \cdot \mathrm{mol}^{-1}$ & -41.921 & 21.672 & 30.71 & -22.37 & 0.10099 \\
$\Delta \eta \cdot 10^{3} / \mathrm{kg} \cdot \mathrm{m}^{-1} \cdot \mathrm{s}^{-1}$ & .1082 & -.4581 & .2747 & .4898 & 0.00505 \\
$\Delta \mathrm{u} / \mathrm{m} \cdot \mathrm{s}^{-1}$ & 212.41 & 171.4 & -176.66 & -176.41 & 0.705352 \\
$\Delta \mathrm{Z} \cdot 10^{-5} / \mathrm{kg} \cdot \mathrm{m}^{-2} \cdot \mathrm{s}^{-1}$ & 7.255 & -0.6415 & -5.3511 & 0.6241 & 0.009398 \\
$\Delta \beta_{\mathrm{s}} 10^{10} / \mathrm{Pa}$ & -1.3593 & 0.4475 & 0.5801 & -0.5587 & 0.022466 \\
$\Delta \mathrm{L}_{\mathrm{f}} \cdot 10^{11} / \mathrm{m}$ & -0.9311 & 0.0954 & 0.5776 & -0.1211 & 0.002131 \\
$\Delta \pi \cdot 10^{-5} / \mathrm{Pa}$ & -0.0372 & -0.6256 & 0.3596 & 0.6213 & 0.000967 \\
$\mathrm{~V}_{\mathrm{f}} \mathrm{E} / \mathrm{m}^{3} \cdot \mathrm{mol}^{-1}$ & 0.0076 & 0.0053 & -0.0104 & -0.005 & 0.000016 \\
\hline
\end{tabular}

Table 12. Parameters of Redlich-Kister equation and standard deviations for benzaldehyde (1) + benzene (2) $\mathrm{T}=308.15 \mathrm{~K}$

\begin{tabular}{lccccc}
\hline \multicolumn{1}{c}{ Functions } & $\mathrm{A}_{0}$ & $\mathrm{~A}_{1}$ & $\mathrm{~A}_{2}$ & $\mathrm{~A}_{3}$ & $\mathrm{~S}$ \\
\hline $\mathrm{V}^{\mathrm{E}} \cdot 10^{6} / \mathrm{m}^{3} \cdot \mathrm{mol}^{-1}$ & -12.97 & 9.7167 & 0.5244 & -4.665 & 0.06366 \\
$\Delta \eta \cdot 10^{3} / \mathrm{kg} \cdot \mathrm{m}^{-1} \cdot \mathrm{s}^{-1}$ & 0.2414 & -0.2507 & 0.0091 & -0.2268 & 0.00292 \\
$\Delta \mathrm{u} / \mathrm{m} \cdot \mathrm{s}^{-1}$ & -232.1 & 73.447 & 14.392 & -59.875 & 1.423618 \\
$\Delta \mathrm{Z} \cdot 10^{-5} / \mathrm{kg} \cdot \mathrm{m}^{-2} \cdot \mathrm{s}^{-1}$ & -1.2923 & -0.627 & 0.156 & 0.0369 & 0.034854 \\
$\Delta \beta_{\mathrm{s}} 10^{10} / \mathrm{Pa}^{-1}$ & 0.2949 & 0.6807 & 0.0688 & -0.6894 & 0.008035 \\
$\Delta \mathrm{L}_{\mathrm{f}} \cdot 10^{11} / \mathrm{m}$ & 0.2679 & 0.188 & -0.002 & -0.1607 & 0.004789 \\
$\Delta \pi \cdot 10^{-5} / \mathrm{Pa}$ & 0.2717 & -0.4804 & 0.0436 & 0.051 & 0.00107 \\
$\mathrm{~V}_{\mathrm{f}} \mathrm{E} / \mathrm{m}^{3} \cdot \mathrm{mol}^{-1}$ & 0.0007 & 0.0069 & -0.0011 & -0.0011 & 0.000028 \\
\hline
\end{tabular}

Table 13. Parameters of Redlich-Kister equation and standard deviations for benzaldehyde (1) + benzene (2) $\mathrm{T}=313.15 \mathrm{~K}$

\begin{tabular}{lccccc}
\hline Functions & $\mathrm{A}_{0}$ & $\mathrm{~A}_{1}$ & $\mathrm{~A}_{2}$ & $\mathrm{~A}_{3}$ & $\mathrm{~S}$ \\
\hline $\mathrm{V}^{\mathrm{E}} \cdot 10^{6} / \mathrm{m}^{3} \cdot \mathrm{mol}^{-1}$ & -13.10 & 14.696 & 0.5644 & -5.4874 & 0.089236 \\
$\Delta \eta .10^{3} / \mathrm{kg}^{-1} \cdot \mathrm{m}^{-1} \cdot \mathrm{s}^{-1}$ & 0.3151 & .1393 & -0.097 & -0.1923 & 0.00288 \\
$\Delta \mathrm{u} / \mathrm{m} \cdot \mathrm{s}^{-1}$ & -183.27 & 110.59 & 12.377 & -65.215 & 1.441009 \\
$\Delta \mathrm{Z} .10^{-5} / \mathrm{kg}^{-} \mathrm{m}^{-2} \cdot \mathrm{s}^{-1}$ & -0.6496 & -0.9651 & 0.1045 & 0.0706 & 0.029793 \\
$\Delta \beta_{\mathrm{s}} 10^{10} / \mathrm{Pa}^{-1}$ & -0.5316 & 0.7407 & 0.1472 & -0.7452 & 0.029421 \\
$\Delta \mathrm{L}_{\mathrm{f}} .10^{11} / \mathrm{m}$ & -0.0311 & 0.2074 & 0.0238 & -0.171 & 0.002137 \\
$\Delta \pi \cdot 10^{-5} / \mathrm{Pa}$ & 0.1927 & -0.2201 & -0.0526 & 0.1178 & 0.000644 \\
$\mathrm{~V}_{\mathrm{f}}{ }^{\mathrm{E}} / \mathrm{m}^{3} \cdot \mathrm{mol}^{-1}$ & 0.0047 & 0.0027 & 0.0004 & -0.0024 & 0.000017 \\
\hline
\end{tabular}

Deviation of physical property of liquid mixtures from the ideal behaviour is the measure of the interaction between the molecules which is attributed to either adhesive or cohesive forces. The variation of excess molar volume with mole fraction of benzaldehyde (1) + benzene (2) at $(303.15,308.15$ and 313.15$) \mathrm{K}$ is represented in Figure 1. 


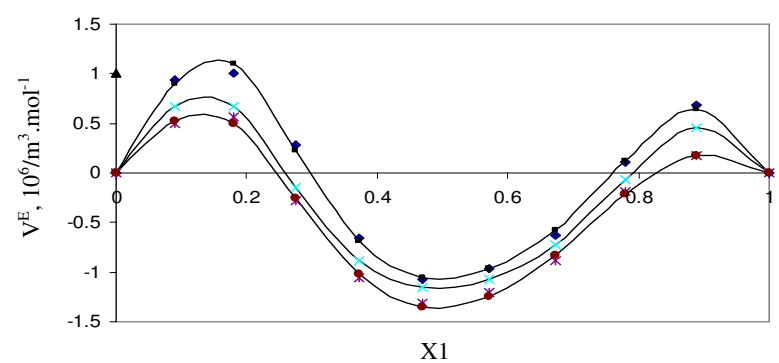

Figure 1. Excess molar volume $\left(\mathrm{V}^{\mathrm{E}}\right)$ for [Benzaldehyde (1) +Benzene (2)]: $\mathrm{T}=303.15 \mathrm{~K}$; $\boldsymbol{\Delta} \mathrm{T}=308.15 \mathrm{~K} ; \boldsymbol{\mathrm { a }}=313.15 \mathrm{~K}$

The excess molar volume shows positive deviation for all the studied temperatures and for any composition ${ }^{11,12}$. The increase in Excess molar volume with increase in benzaldehyde implies that dipole induced dipole interactions are weak owing to the decrease in their polarizability.

The negative deviation in viscosity ${ }^{13}$ over the whole composition range for all mixtures decreases in absolute value as the temperature is increased, due to weakening of interaction (figure.2). This negative deviation suggests that in these mixtures, the forces between unlike molecules are lesser than the forces between like molecules.

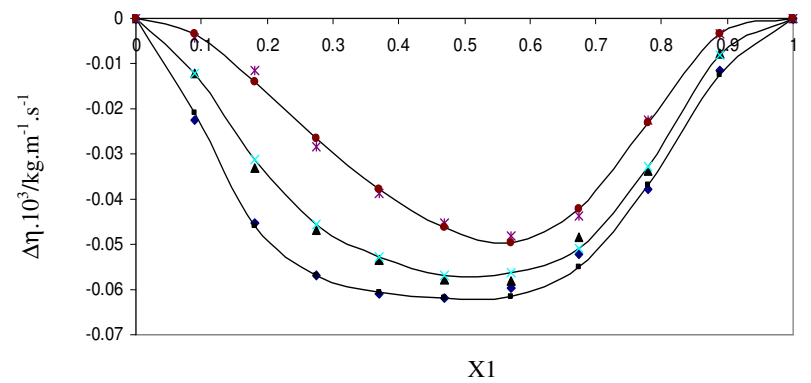

Figure 2. Deviation in viscosity $(\Delta \eta)$ for [Benzaldehyde(1)+Benzene(2)] $\bullet=303.15 \mathrm{~K}$; $\mathrm{T}=308.15 \mathrm{~K} ;: \mathbf{\mathrm { T }}=313.15 \mathrm{~K}$

Speed of sound depends on intermolecular free length. Increase in free length leads to decrease in speed of sound. Negative deviation (Figure 3) indicates that, if the predominant effect in the mixture is structure-breaking property resulting in expansion and the speed of sound through the mixture will be slower. It also suggests weak interactions between unlike molecules

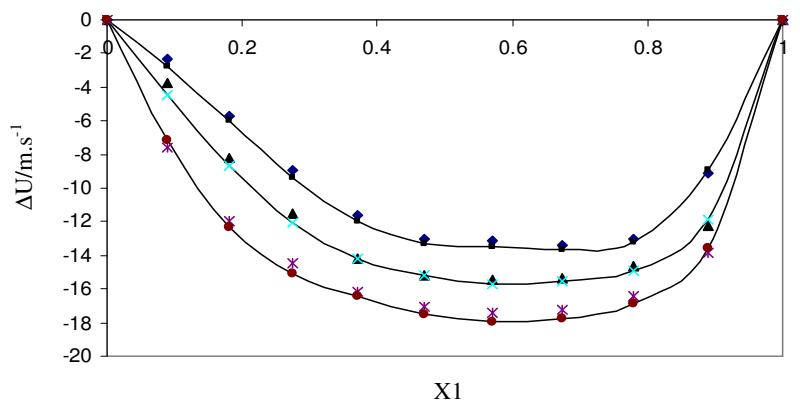

Figure 3. Deviation in speed of sound $(\Delta \mathrm{u})$ for [Benzaldehyde(1)+Benzene(2)] : $\mathrm{T}=303.15 \mathrm{~K}$; $\Delta \mathrm{T}=308.15 \mathrm{~K} ; \mathbf{\square} \mathrm{T}=313.15 \mathrm{~K}$ 
Negative deviation in acoustic impedance (Figure 4) indicates weak dipolar interactions. The magnitude and sign of isentropic compressibility can be used to study the structure making/breaking properties of the liquid mixtures. The positive deviation indicates the structure breaking effect. Positive deviation in isentropic compressibility (Figure 5) reveals that the packing molecules in the mixture is less compact than in the pure components. The positive values indicate the mixture is more compressible than the corresponding ideal mixture.

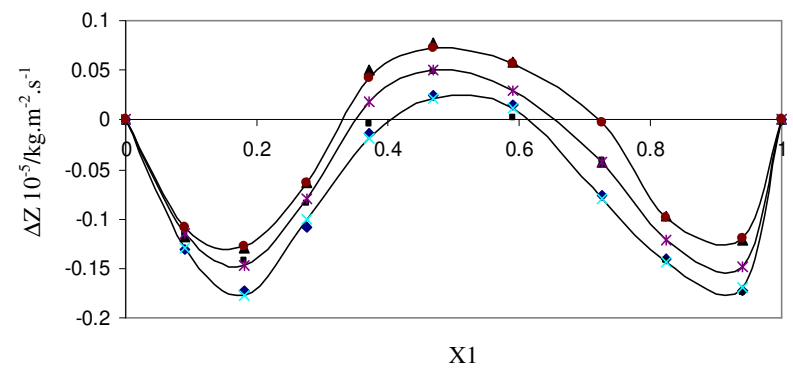

Figure 4. Excess Acoustic impedance $(\Delta \mathrm{Z})$ for [Benzaldehyde (1) +Benzene (2)]: $\mathrm{T}=303.15 \mathrm{~K} ; \boldsymbol{\Delta} \mathrm{T}=308.15 \mathrm{~K} ;: \mathbf{\sim} \mathrm{T}=313.15 \mathrm{~K}$

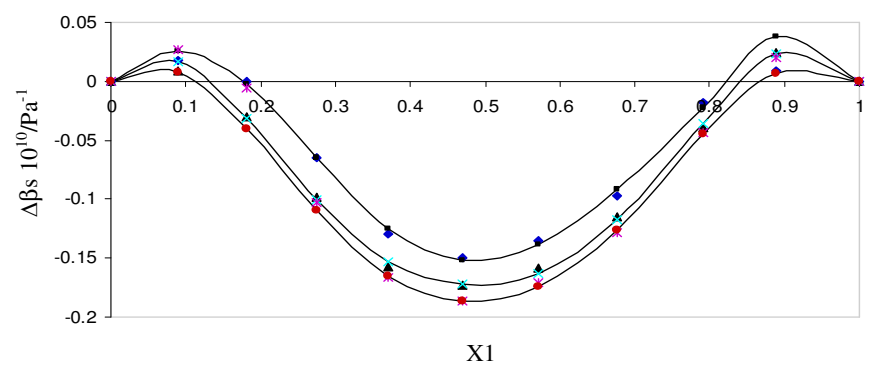

Figure 5. Deviation in isentropic compressibility $(\Delta \beta \mathrm{s})$ for [Benzaldehyde (1) +Benzene (2)]: $\checkmark=303.15 \mathrm{~K} ; \boldsymbol{\Delta} \mathrm{T}=308.15 \mathrm{~K} ; \boldsymbol{\mathrm { T }} \mathrm{T}=313.15 \mathrm{~K}$

The free length (Figure 6) is the distance between the surfaces of the neighbouring molecules. It is used to study the nature and strength of molecular interactions ${ }^{2}$. Positive deviation indicates weak type of interactions involving dispersion forces between unlike molecules, and also indicates that molecules are far away in the system.

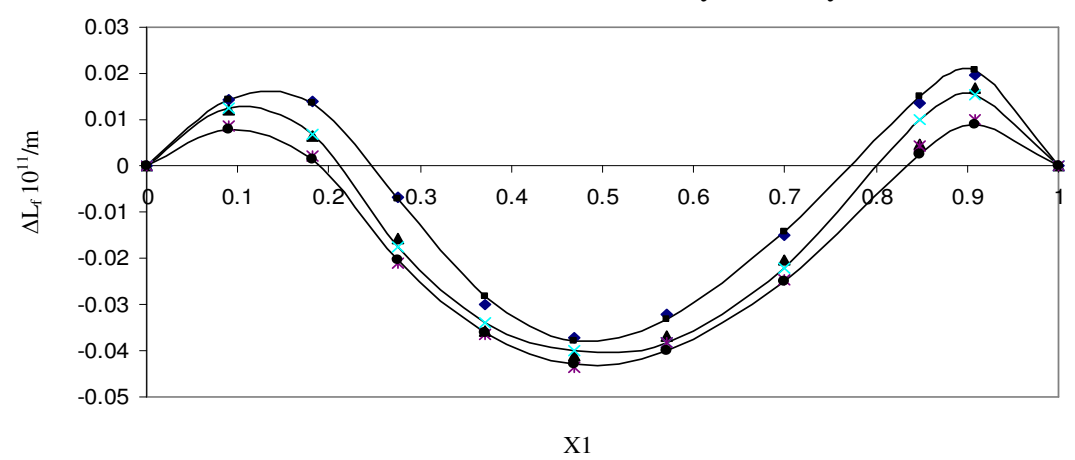

Figure 6. Deviation in intermolecular free length $\left(\Delta \mathrm{L}_{\mathrm{f}}\right)$ for Benzaldehyde(1) +Benzene(2)] : - $\mathrm{T}=303.15 \mathrm{~K} ; \boldsymbol{\Delta} \mathrm{T}=308.15 \mathrm{~K} ; \mathbf{\sim} \mathrm{T}=313.15 \mathrm{~K}$ 
The internal pressure (Figure 7) values of the liquid mixtures decreases and free volume values increase with increase in temperature. At low temperature, Brownian moment is less. However, decrease in internal pressure and increase in free volume (Figure 8) is due to destruction of association at high temperatures. Since cohesive forces get loosened due to thermal energy at high temperatures, the internal pressure value decreases while free volume value increases. The increase in free volume point out to a loosely packing of molecules.

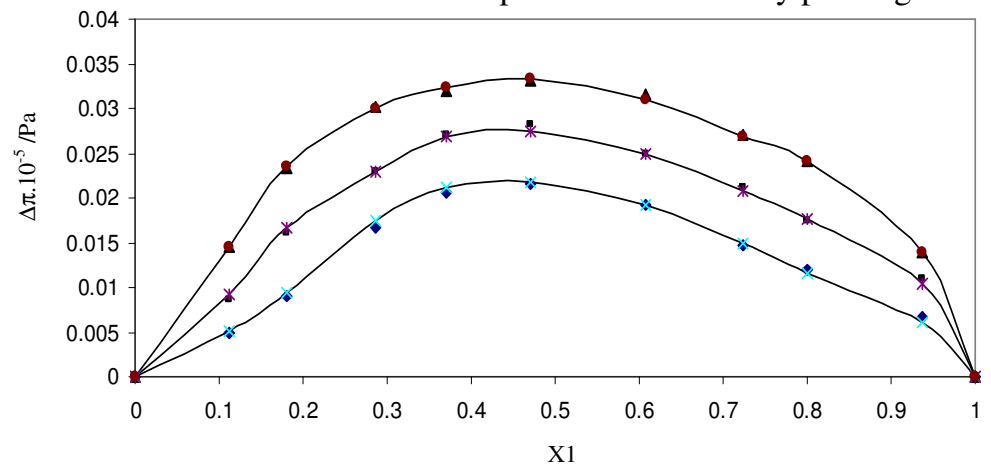

Figure 7. Excess internal pressure $(\Delta \pi)$ for [Benzaldehyde $(1)+$ Benzene $(2)]: ~ T=303.15 \mathrm{~K}$; $\Delta \mathrm{T}=308.15 \mathrm{~K} ; \boldsymbol{\mathrm { T }}=313.15 \mathrm{~K}$

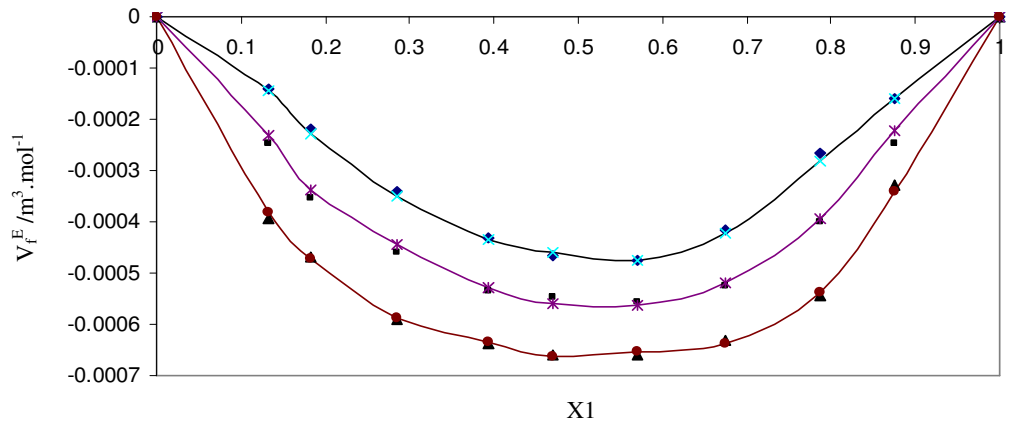

Figure 8. Excess Free volume $\left(\Delta \mathrm{V}_{\mathrm{f}}^{\mathrm{E}}\right)$ for [Benzaldehyde (1) + Benzene (2)]: $\uparrow \mathrm{T}=303.15 \mathrm{~K}$; $\Delta \mathrm{T}=308.15 \mathrm{~K} ; \boldsymbol{\square} \mathrm{T}=313.15 \mathrm{~K}$

\section{Conclusion}

It has been observed that positive deviations were observed for excess molar volumes of the mixture. In the case of viscosity and speed of sound, negative deviations were observed for the benzaldehyde + benzene binary mixture at 303.15, 308.15 and $313.15 \mathrm{~K}$. It has also been observed that positive deviations were observed for excess intermolecular free length, excess isentropic compressibility and negative deviations were observed for excess acoustic impedance, excess internal pressure. It indicates that weak interaction involving dispersion forces between unlike molecules. It has been concluded that the McAllister model is very well suited for correlating the Kinematic viscosity which is indicated by low percentage standard deviation with minimum number of coefficients. It has been concluded that Jouyban - Acree model is very well suited for correlating the viscosity, speed of sound and density of the binary mixture. It is also clear that the Redlich-Kister polynomial equation can represent the excess molar volume, deviations of viscosity and deviation in Speed of Sound very well which is indicated by low standard deviations. 


\section{Acknowledgement}

The authors thank the University authorities for providing the necessary facilities to carry out the work.

\section{References}

1. Ewing M B, Levian B J and Marsh K N, J Chem Thermodyn, 1970, 2, 689.

2. Kenart C M and Kenart W, Phys Chem Liq., 2000, 38, 155-180.

3. Saravanakumar K, Baskaran R and Kubendran T R, J Soln Chem., 2011, 40, 955-967.

4. Saravanakumar K, Baskaran R and Kubendran T R, Asian J Chem., 2011, 23, 2643-2647.

5. Saravanakumar K, Baskaran R and Kubendran T R, Oriental J Chem., 2011, 27, 1073.

6. McAllister R A, AIChE J., 1960, 6, 427-431.

7. Krishnan M R V and Laddha G S, Trans - Indian Inst Chem Eng., 1968, 10, 56-60.

8. Jouyban A, Khoubnasabjafari M, Vaezgharamaleki Z, Fekari Z and Acree W E Jr, Chem Pharm Bull., 2005, 53, 519.

9. Redlich O and Kister A T, Ind Eng Chem., 1948, 40, 345.

10. Riddick J A, Bunger W B and Sakano T K, Organic Solvents. Physical Properties and Methods of Chemistry, $4^{\text {th }}$ Edn., Wiley-Interscience, New York, 1986.

11. Treszczanowicz A J, Kiyohara O and Benson G C, J Chem Thermodyn., 1981, 13, 253.

12. Roux A, Desnoyers J Proc Indian Acad Chem Soc., 1978, 98, 435-439.

13. Fort R J and Moore W R, Trans Faraday Soc., 1966, 62, 1112.

14. Azhagiri S, Jayakumar S, Padmanaban R, Gunasekaran S and Srinivasan S, $J$ Solution Chem., 2009, 38, 441-448.

15. Mahendra Nath Roy, Rajesh Kumar Das and Arijit Bhattacharjee, J Chem Eng Data, 2008, 53(7), 1431.

16. Manapragada V Rathnam, Sudhir Mohite and Manapragada S Kuma, J Chem Engg Data., 2009, 54(2), 305-309.

17. Baskaran R and Kubendran T R, J Chem Eng Data, 2008, 53, 1956. 